\title{
CircMTO1 Attenuated Acute Kidney Injury Through Regulating miR-337
}

\author{
Chuan-chuan Shi, ${ }^{1}$ Lu-yan Pan, ${ }^{2}$ Zhi-yong Peng, ${ }^{1}$ and Jian-guo Li ${ }^{1,3}$
}

\begin{abstract}
Acute kidney injury (AKI) is an independent risk factor for the increased risk of death in patients with sepsis. In the current study, we first investigated the expression of circMTO1 in sepsis-induced AKI, and the underlying mechanism was further elucidated. The results showed that circMTO1 expression level was significantly decreased in serums and kidney tissues of US rats and RMCs treated with LPS. Besides, circMTO1 overexpression promoted cell viability, suppressed cell apoptosis and cytokines production of LPS-treated RMCs. Bioinformatics analysis showed that circMTO1 served as a sponge for miR-337. Furthermore, circMTO1 could inhibit the expression of KLF6. Altogether, our study first reported that circMTO1 expression was decreased in sepsis-induced AKI rat models and RMCs treated with LPS. CircMTO1 overexpression could attenuate AKI development by sponging miR-337 and regulating KLF6 expression, which may provide new ideas for evaluation the pathogenesis and the treatment of sepsis-induced AKI.
\end{abstract}

KEY WORDS: sepsis; AKI; circMTO1; miR-337; KLF6.

\section{INTRODUCTION}

Sepsis is a systemic inflammatory response syndrome caused by pathogenic microorganisms invading the body, which can give rise to septic shock and multiple organ dysfunction syndromes [1]. Sepsis is a common disease in critically ill patients, with a $20-30 \%$ in-hospital mortality 63 rate [2]. Kidney is one of the most sensitive organs which can be involved in sepsis. And acute kidney injury (AKI) is an independent predictor of mortality of patients with sepsis $[3,4]$. Despite extensive advancements in

\footnotetext{
${ }^{1}$ Department of Intensive Care Unit, Zhongnan Hospital of Wuhan University, Wuhan, 430071, Hubei, China

${ }^{2}$ Henan Health Cadre College, Zhengzhou, 450000, Henan, China

${ }^{3}$ To whom correspondence should be addressed at Department of Intensive Care Unit, Zhongnan Hospital of Wuhan University, Wuhan, 430071, Hubei, China. E-mail: lijianguo_znhwu@163.com
}

sepsis-induced AKI therapeutic strategies, the prognosis of patients still remains unfavorable [5]. Thus, it is imperative to explore the molecular mechanisms underlying sepsis-induced AKI.

Compared with regular linear RNAs, circRNAs (circRNAs) exhibit the notable characteristic of covalently closed loop structures with neither $5^{\prime}$ caps nor $3^{\prime}$ polyadenylated tails, leading to a stable structure and high tissue-specific expression [6,7]. Abnormal circRNAs expression has been detected in the development of several diseases, such as atherosclerosis [8], myocardial infarction [9], and nervous system disorders [10]. Until now, few studies focused on the role of circRNAs in the progression of sepsisinduced AKI. CircMTO1 (mitochondrial translation optimization 1 homolog, circ_0007874) has been demonstrated to play an inhibitory role in some kinds 
of tumor development. However, the molecular function of circMTO1 in sepsis-induced AKI was still unclear.

MicroRNAs (miRNAs) belong to noncoding singlestranded RNAs which are involved in many physiological processes like cell proliferation, apoptosis, and migration [11]. Previous studies have reported the association between microRNAs and AKI [12]. Nevertheless, the role of miR-337 in AKI was not yet elucidated. In the present study, we first investigated the effect of circMTO1 on the progression of sepsis-induced AKI, and the underlying mechanism was further elucidated.

\section{MATERIALS AND METHODS}

\section{Establishment of the US Model}

Adult male Sprague-Dawley (SD) rats weighing 230 $\pm 10 \mathrm{~g}$ were obtained from Beijing Laboratory Animal Research Center (Beijing, China). The rats were housed under standard environmental conditions (temperature 25 $\pm 2{ }^{\circ} \mathrm{C}$, humidity $55 \pm 5 \%$, and 12 -h light $/ 12$-h dark cycle) and given free access to food and tap water. All rats were randomly divided two groups with 6 in each group and then anesthetized via intraperitoneal injection of chloral hydrate $(10 \%, 0.3 \mathrm{ml} / \mathrm{kg})$. The rats were fixed in a supine position, and the incision was then made along the left rectus muscle. In urine-derived sepsis (US) group, the left ureter is incised and ligated in the middle section, resulting in acute upper urinary obstruction. An Escherichia coli suspension (ATCC 25922, $1 \times 10^{8} / \mathrm{ml}, 0.5 \mathrm{ml} / \mathrm{kg}$ ) was then injected into the distal ureter as previously described [13]. In the sham group, the left ureter was only separated and the incision with sutured. All rats were sacrificed $24 \mathrm{~h}$ after the surgery. The $5 \mathrm{ml}$ serum samples from these rats were centrifuged at $12000 \mathrm{~g}$ for $3 \mathrm{~min}$, and the supernatant was frozen at liquid nitrogen, and the harvested left kidney tissues were stored at liquid nitrogen for further analysis. This study was carried out with the approval of the Institutional Animal Care and Use Committee of Tianjin Medical University.

\section{Cell Culture and Transfection}

Rat mesangial cells (RMCs, purchased from the Cell Bank of the Chinese Academy) were maintained in Dulbecco's modified Eagle's medium containing 10\% fetal bovine serum and $1 \%$ penicillin/streptomycin under an atmosphere of a humidified air and $5 \% \mathrm{CO}_{2}$ at $37^{\circ} \mathrm{C}$. Until around $60 \%$ of confluence, RMCs cells were treated with
$100 \mathrm{ng} / \mathrm{mL}$ LPS for $24 \mathrm{~h}$ to induce injury inflammation. Cells were seeded in 6-well plates and then transfected with si-circMTO1, miR-337 inhibitor, or their corresponding controls mixed with lipofectamine 2000 reagent according to the manufacturer's protocols.

\section{Cell Viability}

RMCs $\left(1 \times 10^{3}\right.$ cells/well) with different transfections were seeded in 96-well plates and cultured for $0,24,48,72$, $96 \mathrm{~h}$. After being washed three times with PBS, cells were treated with $500 \mu \mathrm{g} / \mathrm{mL}$ of MTT solution for $3 \mathrm{~h}$. Subsequently, $200 \mu$ of dimethylsulfoxide (DMSO) was added to dissolve precipitates. The optical density (OD) of each well was measured at $490 \mathrm{~nm}$ under a microplate spectrophotometer.

\section{Cell Apoptosis}

The cells were suspended in the binding buffer, fixed in ice-cold 70\% ethanol, and stained with FITC/Annexin V and PI in the dark at room temperature for $15 \mathrm{~min}$. The apoptosis detection was performed by flow cytometry assay (FACScan, BD Biosciences, San Jose, CA, USA) and analyzed using CellQuest software.

\section{Enzyme Linked Immunosorbent Assay}

According to the manufacturer's instruction, the concentration of inflammatory cytokines in serum (TNF- $\alpha$, IL$1 \beta$ and IL-6) was determined using an ELISA Kit. Blood urea nitrogen (BUN) and serum creatinine were determined using a Urea Nitrogen Colorimetric Detection Kit and Creatinine Urinary Detection Kit (Invitrogen, CA, USA).

\section{Real Time Quantitative PCR Analysis}

Total RNA from kidney tissues and RMCs was isolated using TRIzol reagent (Invitrogen) following the manufacturer's protocol. And cDNA synthesis was conducted using the TaqMan MicroRNA Reverse Transcription Kit (Applied Biosystems) for miR-337 and using One Step PrimeScript cDNA Kit (Qiagen) for circMTO1 and KLF6. RT-PCR was performed using the SYBR ${ }^{\circledR}$ Premix Ex Taq ${ }^{\mathrm{TM}}$ II (Takara) and the Applied Biosystems 7500 Real-time PCR System (Applied Biosystem). The relative expression was analyze by using $2-\Delta \Delta \mathrm{CT}$ method and normalized to GAPDH or U6. The primers were as follows: circMTO1 forward 5'- TTACCAGCCGAGTA GAGTTCC-3' and reverse 5'- ATCCATTCCTTCAG GTTCCAAC-3'; miR-630 forward 5'- CGCTTCAG 
CTCCTATATGA-3' and reverse 5'- GTGCAGGG TCCGAGGT-3'; lnc-NC 5'-UGGACAACAUGGGC UCU-3'; KLF6 forward 5'-TCAAATGCTATCCC CTTTCC-3' and reverse $5^{\prime}$ - CCAGGGCTAGGAAGTAGGAG-3'; U6 forward 5'-GCTCGCTTCGGCAGCACA-3' and reverse 5'-GAGGTA TTCGCA CCAGAG GA-3'; and GAPDH forward 5'- TGTGGGCATCAATGGATTTGG$3^{\prime}$ and reverse 5'- ACACCATGTATTCCGGGTCAAT-3'.

\section{Western Blotting Analysis}

Proteins were extracted from kidney tissues and cultured RMCs by RIPA buffer (Sigma-Aldrich; Merck $\mathrm{KGaA}$ ) containing a mixture of protease inhibitors. Equal quantities of protein were separated via SDS-PAGE on a $10 \%$ gel and transferred to PVDF membranes. After blocking with 5\% skimmed milk, the membrane was incubated with the primary antibodies anti-KLF6 at $4{ }^{\circ} \mathrm{C}$ overnight, followed by incubating with anti-rabbit horseradish peroxidase-conjugated secondary antibody and detected using enhanced chemiluminescence detection system.

\section{Luciferase Reporter Assay}

The fragment of circMTO1 obtaining wild type (WT) or Mutant (Mut) miR-337 binding sites was constructed by Genomeditech (Shanghai, China) and inserted into pGL3 Basic vector. Cells were seeded into 24-well plates and cotransfected with the luciferase reporter constructs, miR-337 inhibitor, and Renilla luciferase construct (Promega) and incubated for $24 \mathrm{~h}$. The relative luciferase activities were measured using the Dual-Luciferase Reporter System (Promega).

\section{Statistical Analysis}

All measurement data were expressed as the mean \pm standard deviation. Differences were calculated with Student's $t$ test or one-way ANOVA. All statistical analyses were performed using the SPSS 17.0 software and GraphPad Prism 6. A value of $P<0.05$ was considered to indicate a statistically significant difference.

\section{RESULTS}

\section{CircMTO1 Expression Was Decreased in Serum and Kidney Tissue of US Rats}

To evaluate the role of circMTO1 in sepsis-AKI, we first measured circMTO1 expression in serum and kidney tissues of US rats using RT-qPCR. The result showed that
circMTO1 expression level was significantly downregulated in serums and kidney tissues of US rats in comparison with that in control rats (Fig. 1a). As expected, renal function indicators (BUN and serum creatinine) were upregulated in US rats (Fig. 1b). Besides, the concentration of inflammation cytokines (TNF- $\alpha$, IL-1 $\beta$ and IL-6) was significantly higher in US rats than those in control rats (Fig. 1c).

\section{CircMTO1 Overexpression Promoted Cell Viability, Suppressed Cell Apoptosis, and Cytokines Production of LPS-Treated RMCs}

Next we explored the role of circMTO1 in LPStreated RMCs. After treated with LPS, the expression of circMTO1 was notably decreased in RMCs (Fig. 2a). And then circMTO1 overexpression was established in LPS-treated RMCs by transfection (Fig. 2b). MTT assays revealed that circMTO1 mimic accelerated the proliferation of LPS-treated RMCs significantly (Fig. 2c). And flow cytometry analysis demonstrated that cell apoptosis rate was lower in LPS-treated RMCs after transfection with circMTO1 mimic (Fig. 2d). In addition, circMTO1 overexpression could obviously downregulated the expression of related cytokines (Fig. 2e).

\section{CircMTO1 Serves as a Sponge for miR-337}

Given that circRNA has been shown to act as miRNA sponge [14], we further predicted the potential circRNAmiRNA interactions using CircInteractome (https://circinteractome.nia.nih.gov/). As shown in Fig. 3 a, miR-337 was observed to have a potential binding site with circMTO1. Luciferase reporter assay verified that miR337 mimic suppressed the luciferase activity of only circMTO1 wild type in cells (Fig. 3b). And miR-337 expression was significantly upregulated in US rat models and LPS-treated RMCs (Fig. 3c). Under LPS-stimulated condition, circMTO1 overexpression could downregulated miR-337 level significantly in RMCs, which was reversed by miR-337 mimic (Fig. 3d). MTT assay demonstrated that miR-337 mimic could repress the induction by circMTO1 overexpression in the proliferation of RMCs treated with LPS at $96 \mathrm{~h}$ (Fig. 3e). Besides, miR-337 mimic could increase the apoptosis rate of LPS-treated RMCs transfected with circMTO1 mimic (Fig. 3f). As expected, miR-337 mimic obviously promoted cytokines production in cells transfected with circMTO1 mimic (Fig. 3g). 

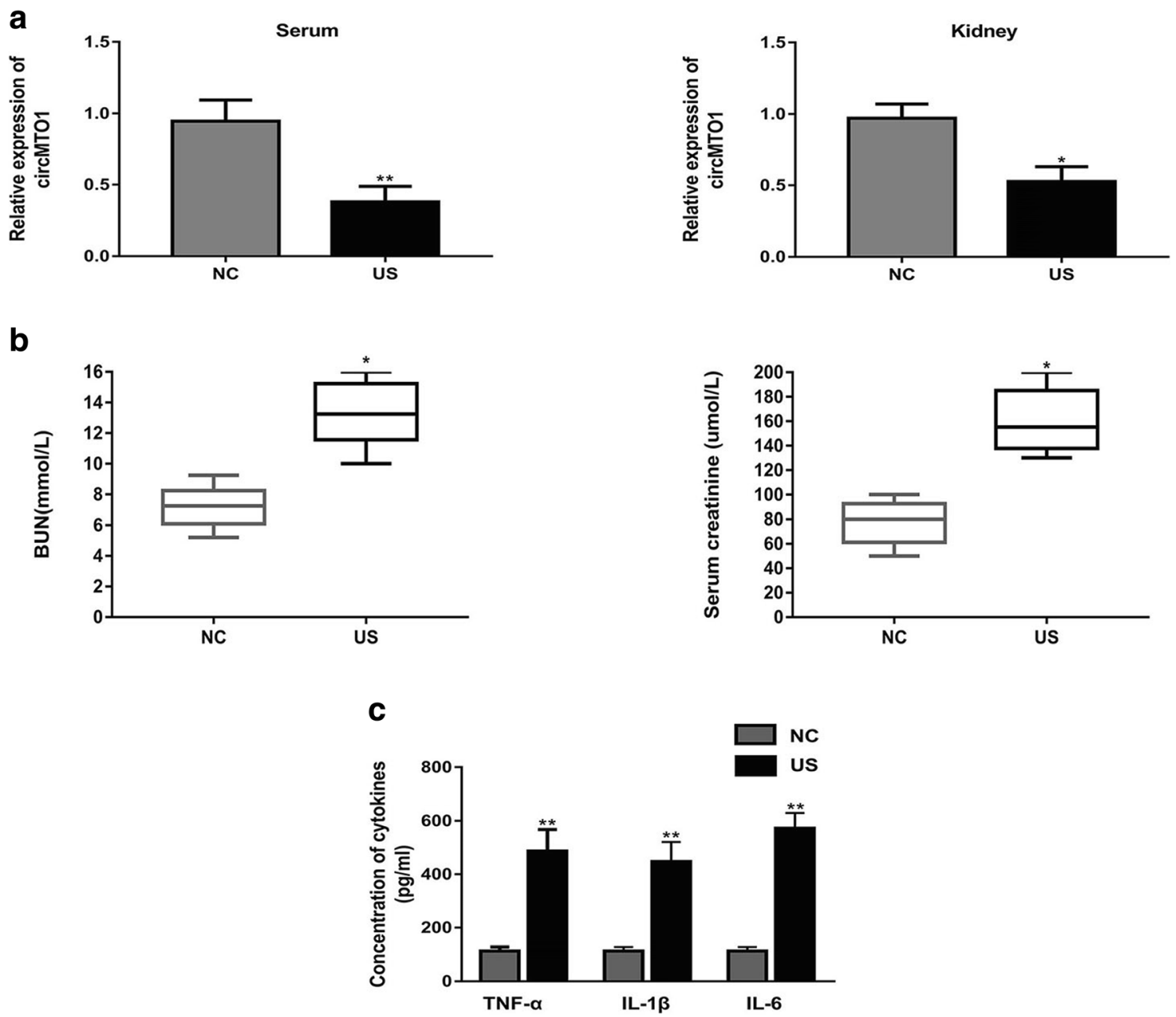

Fig. 1. CircMTO1 expression was decreased in serum and kidney tissue of US rats. a circMTO1 expression level was significantly downregulated in serums and kidney tissues of US rats in comparison with that in control rats. b BUN and serum creatinine) were significantly upregulated in US rats. $\mathbf{c}$ The concentration of inflammation cytokines (TNF- $\alpha$, IL-1 $\beta$, and IL-6) was significantly higher in US rats than those in control rats. ${ }^{*} P<0.05$, ${ }^{* * *} P<0.01$ compared to control group.

\section{CircMTO1 Overexpression Could Inhibit the Expression of KLF6}

KLF6 has been confirmed to have a potential role in inflammatory response [15]. Subsequently, we analyzed the effect of circMTO1 on the expression of KLF6 in LPStreated RMCs. The result showed that KLF6 expression level was significantly upregulated in US rat models and RMCs treated with LPS (Fig. 4a, b). Meanwhile, KLF6 expression was positively correlated with circMTO1 expression in kidney tissues (Fig. 4c). Further, circMTO1 overexpression could suppress the expression of KLF6 obviously, which was reversed by miR-337 mimic (Fig. 4d).

\section{DISCUSSION}

Up to now, circRNA have been reported to participate in many crucial biological events such as cell proliferation, apoptosis, metastasis, and differentiation $[16,17]$. A growing number of studies have shown that aberrant expression of circRNA plays a regulatory role in different diseases via different mechanisms [18-20]. In addition, circRNAs may have potential as clinical diagnostic markers and therapeutic targets in sepsis $[21,22]$. Here, we first explored the role of circMTO1 in sepsis-AKI model. The results showed circMTO1 expression level was 

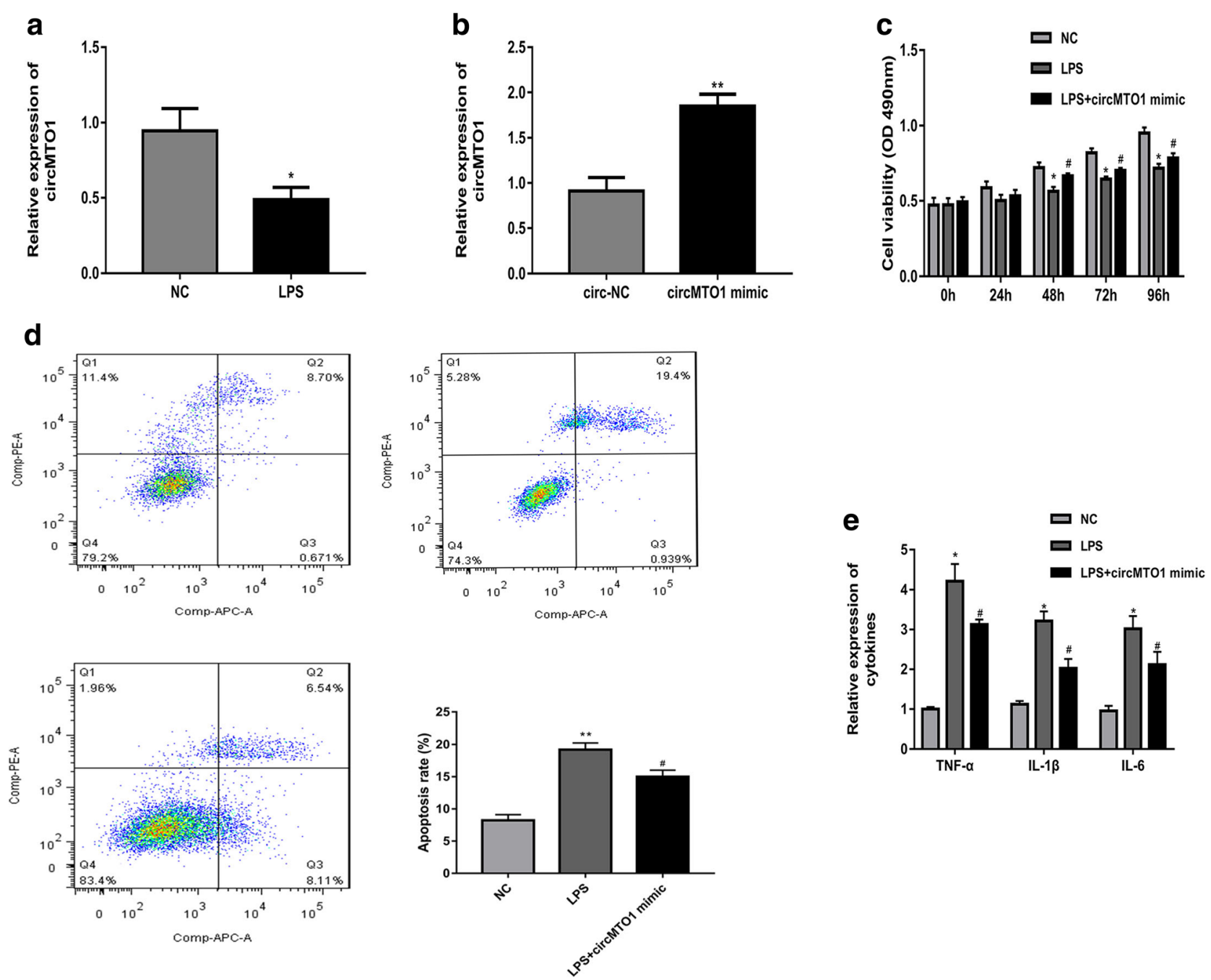

Fig. 2. CircMTO1 overexpression promoted cell viability, suppressed cell apoptosis and cytokines production of LPS-treated RMCs. a: The expression of circMTO1 was notably decreased in RMCs treated with LPS. b circMTO1 overexpression was established in LPS-treated RMCs by transfection. $\mathbf{c}$ MTT assays revealed circMTO1 mimic accelerated the proliferation of LPS-treated RMCs significantly. $\mathbf{d}$ Flow cytometry revealed that cell apoptosis rate was lower in LPS-treated RMCs after transfection with circMTO1 mimic. e: circMTO1 overexpression could obviously downregulated the expression of related cytokines. ${ }^{*} P<0.05,{ }^{* *} P<0.01$ compared to control group; ${ }^{\#} P<0.05$ compared to LPS group.

significantly decreased in serums and kidney tissues of US rats. Meanwhile, the concentration of inflammation cytokines (TNF- $\alpha$, IL-1 $\beta$, and IL-6) was significantly higher in US rats. And then we observed that circMTO1 overexpression could promote cell viability and inhibited cell apoptosis and cytokines production of LPS-treated RMCs. These data implied circMTO1 mimic could suppress the biological behavior of RMCs under LPS-stimulated condition.

Given that circRNAs could regulate mRNA translation indirectly by competing for miRNAs [23], we predicted that circMTO1 functionally act as the sponge for miR-337 through miRNA screening. Previous research has demonstrated that suppressing miR-337 could attenuate inflammation progression in some diseases $[24,25]$. Likewise, miR337 expression level was significantly upregulated in US rat models and RMCs cells treated with LPS. The recovery experiment revealed that miR-337 mimic could reverse the inhibitory effect on inflammation of circMTO1. Krüppel-like factors (KLFs) are highly conserved zinc-finger proteins that regulate cellular transcription [26]. KLF6 has been reported to participate in proinflammatory gene expression in kidney 
a

$$
\begin{aligned}
\text { circMT01 WT } & 5^{\prime} \ldots . . . . \text { UGAGAGCUCAGAUUGAUAGGAAA ......3 } \\
\text { miR-337 } & 3^{\prime}, \ldots . . . \text { CUUCUUUCCGUAGUAUAUCCUC }
\end{aligned}
$$

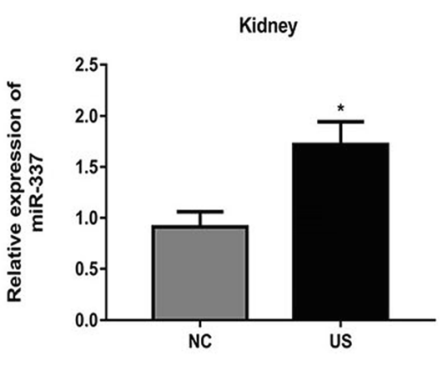

$\mathbf{f}$
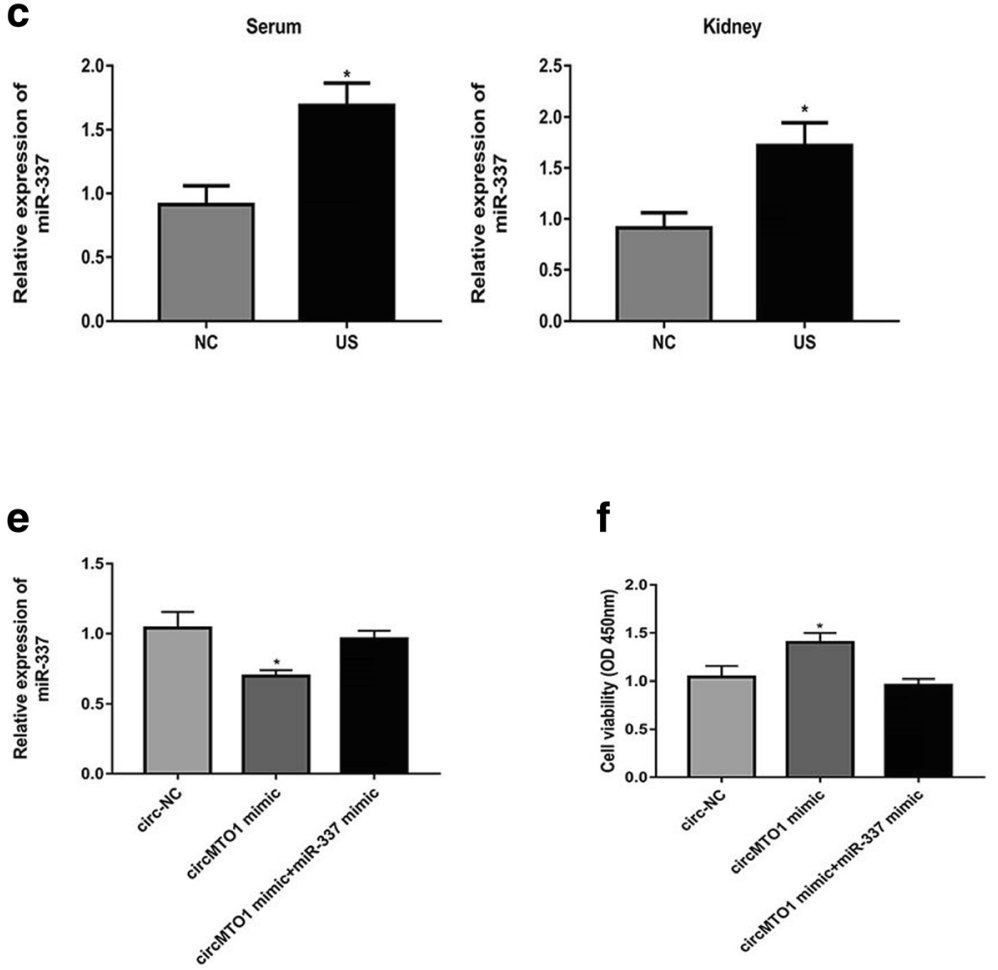

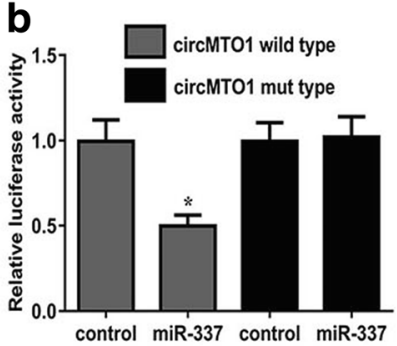

d

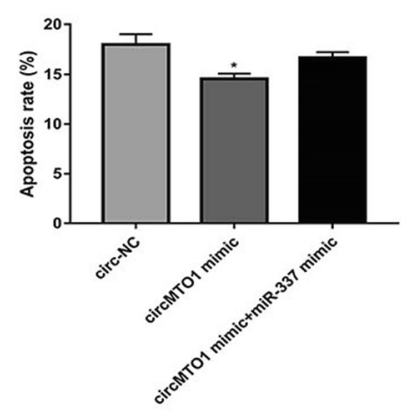

g

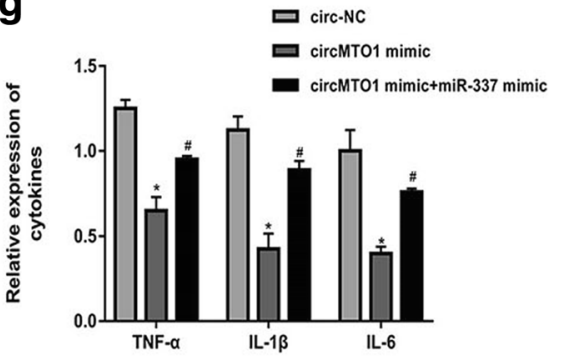

Fig. 3. CircMTO1 serves as a sponge for miR-337. a miR-337 was observed to have a potential binding site with circMTO1. b miR-337 mimic suppressed the luciferase activity of only circMTO1 wild type in cells. $\mathbf{c}$ miR-337 expression was significantly upregulated in US rat models and LPS-treated RMCs. $\mathbf{d}$ circMTO1 overexpression could downregulated miR-337 level significantly in RMCs, which was reversed by miR-337 mimic. e MTT assay demonstrated that miR-337 mimic could repress the induction by circMTO1 overexpression in the proliferation of RMCs treated with LPS at $96 \mathrm{~h}$. f miR-337 mimic could increase the apoptosis rate of LPS-treated RMCs transfected with circMTO1 mimic. $\mathbf{g}$ miR-337 mimic obviously promoted cytokines production in cells transfected with circMTO1 mimic. ${ }^{*} P<0.05$ compared to control group; ${ }^{\#} P<0.05$ compared to circMTO1 mimic group.

diseases [27]. We noticed that KLF6 expression was significantly upregulated in US rats and RMCs. Besides, circMTO1 overexpression could suppress the expression of KLF6 obviously, which was reversed by miR-337 mimic. These data suggested that circMTO1 may function through regulation of miR337/KLF6 axis in AKI-sepsis progression.

\section{CONCLUSION}

Altogether, we first discovered that circMTO1 expression was decreased in sepsis-induced AKI rat models and RMCs treated with LPS. CircMTO1 overexpression could attenuate AKI development by sponging miR-337 and regulating KLF6 expression, which may provide new 




C



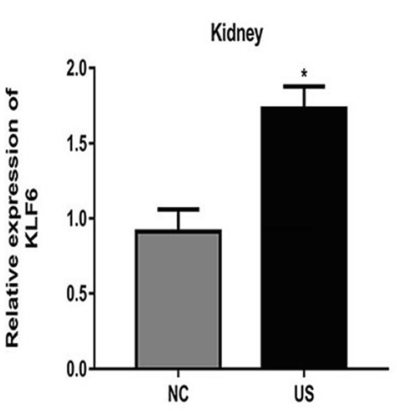

d

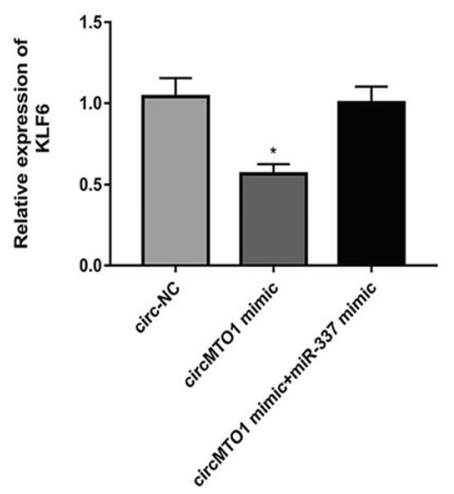

b
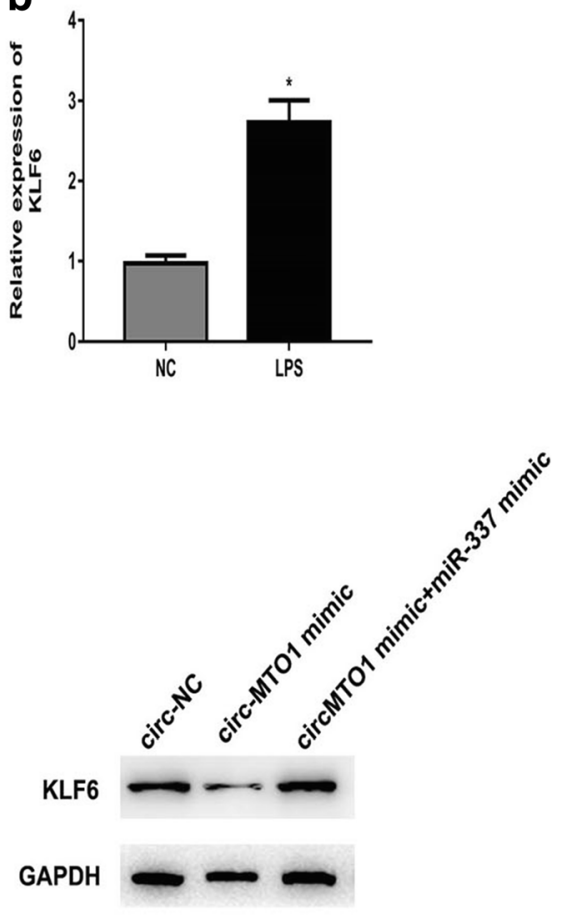

Fig. 4. CircMTO1 overexpression could inhibit the expression of KLF6. a KLF6 expression level was significantly upregulated in US rats' models. b KLF6 expression level was significantly upregulated in RMCs treated with LPS. c KLF6 expression was positively correlated with circMTO1 expression in kidney tissues. d circMTO1 overexpression could suppress the expression of KLF6 obviously, which was reversed by miR-337 mimic at both mRNA and protein levels. ${ }^{*} P<0.05$ compared to control group.

ideas for the evaluation of the pathogenesis and the treatment of sepsis-induced AKI.

\section{FUNDING INFORMATION}

This work was supported by the National Natural Science Foundation of China (grant number: No.81571946).

\section{COMPLIANCE WITH ETHICAL STANDARDS}

Conflict of Interest. All authors declare that they have no conflicts of interest.
Open Access This article is licensed under a Creative Commons Attribution 4.0 International License, which permits use, sharing, adaptation, distribution and reproduction in any medium or format, as long as you give appropriate credit to the original author(s) and the source, provide a link to the Creative Commons licence, and indicate if changes were made. The images or other third party material in this article are included in the article's Creative Commons licence, unless indicated otherwise in a credit line to the material. If material is not included in the article's Creative Commons licence and your intended use is not permitted by statutory regulation or exceeds the permitted use, you will need to obtain permission directly from the copyright holder. To view a copy of this licence, visit http://creativecommons.org/licenses/by/4.0/. 


\section{REFERENCES}

1. Singer, M., C.S. Deutschman, C.W. Seymour, M. Shankar-Hari, D. Annane, M. Bauer, R. Bellomo, G.R. Bernard, J.D. Chiche, C.M. Coopersmith, R.S. Hotchkiss, M.M. Levy, J.C. Marshall, G.S. Martin, S.M. Opal, G.D. Rubenfeld, T. van der Poll, J.L. Vincent, and D.C. Angus. 2016. The third international consensus definitions for sepsis and septic shock (Sepsis-3). JAMA 315 (8): 801-810. https:// doi.org/10.1001/jama.2016.0287.

2. Angus, D.C., W.T. Linde-Zwirble, J. Lidicker, G. Clermont, J. Carcillo, and M.R. Pinsky. 2001. Epidemiology of severe sepsis in the United States: Analysis of incidence, outcome, and associated costs of care. Critical Care Medicine 29 (7): 1303-1310. https:// doi.org/10.1097/00003246-200107000-00002.

3. Lopes, J.A., P. Fernandes, S. Jorge, C. Resina, C. Santos, A. Pereira, J. Neves, F. Antunes, and A. Gomes da Costa. 2010. Long-term risk of mortality after acute kidney injury in patients with sepsis: A contemporary analysis. BMC Nephrology 11: 9. https://doi.org/ 10.1186/1471-2369-11-9.

4. Plataki, M., K. Kashani, J. Cabello-Garza, F. Maldonado, R. Kashyap, D.J. Kor, O. Gajic, and R. Cartin-Ceba. 2011. Predictors of acute kidney injury in septic shock patients: An observational cohort study. Clinical Journal of the American Society of Nephrology 6 (7): 1744-1751. https://doi.org/10.2215/CJN.05480610.

5. Angus, D.C. 2011. The search for effective therapy for sepsis: Back to the drawing board? JAMA 306 (23): 2614-2615. https://doi.org/ 10.1001/jama.2011.1853.

6. Hentze, M.W., and T. Preiss. 2013. Circular RNAs: splicing's enigma variations. The EMBO Journal 32 (7): 923-925. https://doi.org/ 10.1038/emboj.2013.53.

7. Vicens, Q., and E. Westhof. 2014. Biogenesis of circular RNAs. Cell 159 (1): 13-14. https://doi.org/10.1016/j.cell.2014.09.005.

8. Holdt, L.M., A. Stahringer, K. Sass, G. Pichler, N.A. Kulak, W. Wilfert, A. Kohlmaier, A. Herbst, B.H. Northoff, A. Nicolaou, G. Gäbel, F. Beutner, M. Scholz, J. Thiery, K. Musunuru, K. Krohn, M. Mann, and D. Teupser. 2016. Circular non-coding RNA ANRIL modulates ribosomal RNA maturation and atherosclerosis in humans. Nature Communications 7: 12429. https://doi.org/ 10.1038/ncomms 12429 .

9. Garikipati, V.N.S., S.K. Verma, Z. Cheng, D. Liang, M.M. Truongcao, M. Cimini, Y. Yue, G. Huang, C. Wang, C. Benedict, Y. Tang, V. Mallaredy, J. Ibetti, L. Grisanti, S.M. Schumacher, E. Gao, S. Rajan, J.E. Wilusz, D. Goukassian, S.R. Houser, W.J. Koch, and R. Kishore. 2019. Circular RNA CircFndc3b modulates cardiac repair after myocardial infarction via FUS/VEGF-A axis. Nature Communications 10 (1): 4317. https://doi.org/10.1038/s41467-019-11777-7.

10. You, X., I. Vlatkovic, A. Babic, T. Will, I. Epstein, G. Tushev, G. Akbalik, M. Wang, C. Glock, C. Quedenau, X. Wang, J. Hou, H. Liu, W. Sun, S. Sambandan, T. Chen, E.M. Schuman, and W. Chen. 2015. Neural circular RNAs are derived from synaptic genes and regulated by development and plasticity. Nature Neuroscience 18 (4): 603-610. https://doi.org/10.1038/nn.3975.

11. Bartel, D.P. 2004. MicroRNAs: Genomics, biogenesis, mechanism, and function. Cell 116 (2): 281-297. https://doi.org/10.1016/s00928674(04)00045-5.

12. Jones, T.F., S. Bekele, M.J. O'Dwyer, and J.R. Prowle. 2018. MicroRNAs in acute kidney injury. Nephron 140 (2): 124-128. https:// doi.org/10.1159/000490204.
13. Shen, J., J. Zhang, X. Jiang, H. Wang, and G. Pan. 2018. LncRNA HOX transcript antisense RNA accelerated kidney injury induced by urinederived sepsis through the miR-22/high mobility group box 1 pathway. Life Sciences 210: 185-191. https://doi.org/10.1016/j.lfs.2018.08.041.

14. Hansen, T.B., T.I. Jensen, B.H. Clausen, J.B. Bramsen, B. Finsen, C.K. Damgaard, and J. Kjems. 2013. Natural RNA circles function as efficient microRNA sponges. Nature 495 (7441): 384-388. https://doi.org/10.1038/nature11993.

15. Date, D., R. Das, G. Narla, D.I. Simon, M.K. Jain, and G.H. Mahabeleshwar. 2014. Kruppel-like transcription factor 6 regulates inflammatory macrophage polarization. The Journal of Biological Chemistry 289 (15): 10318-10329. https://doi.org/10.1074/jbc.M113.526749.

16. Chen, L.L., and L. Yang. 2015. Regulation of circRNA biogenesis. RNA Biology 12 (4): 381-388. https://doi.org/10.1080/ 15476286.2015.1020271.

17. Lasda, E., and R. Parker. 2014. Circular RNAs: Diversity of form and function. RNA 20 (12): 1829-1842. https://doi.org/10.1261/ rna.047126.114.

18. Du, W.W., L. Fang, W. Yang, N. Wu, F.M. Awan, Z. Yang, and B.B. Yang. 2017. Induction of tumor apoptosis through a circular RNA enhancing Foxo3 activity. Cell Death and Differentiation 24 (2): 357-370. https://doi.org/10.1038/cdd.2016.133.

19. Rybak-Wolf, A., C. Stottmeister, P. Glazar, M. Jens, N. Pino, S. Giusti, M. Hanan, et al. 2015. Circular RNAs in the mammalian brain are highly abundant, conserved, and dynamically expressed. Molecular Cell 58 (5): 870-885. https://doi.org/10.1016/j.molcel.2015.03.027.

20. Wu, Y., Y. Zhang, and J.J. Wang. 2017. CircRNA hsa_circ_0005105 upregulates NAMPT expression and promotes chondrocyte extracellular matrix degradation by sponging miR-26a. Cell Biology International 41 (12): 1283-1289. https://doi.org/10.1002/cbin.10761.

21. Dai, Y., Z. Liang, Y. Li, C. Li, and L. Chen. 2017. Circulating long noncoding RNAs as potential biomarkers of sepsis: A preliminary study. Genetic Testing and Molecular Biomarkers 21 (11): 649-657. https://doi.org/10.1089/gtmb.2017.0061.

22. Huang, Q., C. Huang, Y. Luo, F. He, and R. Zhang. 2018. Circulating lncRNA NEAT 1 correlates with increased risk, elevated severity and unfavorable prognosis in sepsis patients. The American Journal of Emergency Medicine 36 (9): 1659-1663. https://doi.org/10.1016/ j.ajem.2018.06.008.

23. Han, B., J. Chao, and H. Yao. 2018. Circular RNA and its mechanisms in disease: From the bench to the clinic. Pharmacology \& Therapeutics 187: 31-44. https://doi.org/10.1016/j.pharmthera.2018.01.010.

24. Mar-Aguilar, F., J.A. Mendoza-Ramirez, I. Malagon-Santiago, P.K. Espino-Silva, S.K. Santuario-Facio, P. Ruiz-Flores, C. RodriguezPadilla, and D. Resendez-Perez. 2013. Serum circulating microRNA profiling for identification of potential breast cancer biomarkers. Disease Markers 34 (3): 163-169. https://doi.org/10.3233/DMA120957.

25. Xia, P., X. Gao, L. Duan, W. Zhang, and Y.F. Sun. 2018. Mulberrin (Mul) reduces spinal cord injury (SCI)-induced apoptosis, inflammation and oxidative stress in rats via miroRNA-337 by targeting Nrf-2. Biomedicine \& Pharmacotherapy 107: 1480-1487. https:// doi.org/10.1016/j.biopha.2018.07.082.

26. Kaczynski, J., T. Cook, and R. Urrutia. 2003. Sp1- and Kruppel-like transcription factors. Genome Biology 4 (2): 206. https://doi.org/ 10.1186/gb-2003-4-2-206.

27. Rane, M.J., Y. Zhao, and L. Cai. 2019. Krupsilonppel-like factors (KLFs) in renal physiology and disease. EBioMedicine 40: 743-750. https://doi.org/10.1016/j.ebiom.2019.01.021.

Publisher's Note Springer Nature remains neutral with regard to jurisdictional claims in published maps and institutional affiliations. 